\title{
Digital Application of Special Effect in Visual Design
}

\section{Gao Xin}

\author{
School of Arts and Communication, Bohai University, Jinzhou, China
}

Keywords: Visual design; Special effect; Digitalization

\begin{abstract}
The application and popularization of computer technology have promoted the development in all fields of the society and the application of computer digital processing technology in film and television shooting is a major innovation in the film and television industry. Film and television producers, by virtue of the digital technology of special effect, have created many classical works, greatly satisfying the audio-visual experience of people. This paper, firstly, describes significant value of digital application of special effect, then analyzes the digital technology application from three aspects, and gives an analysis of digital special effect in visual design, which hopefully can provide reference for related researches on special effect.
\end{abstract}

\section{Introduction}

In recent years, with the rapid economic and social development, the material life of people has been greatly enhanced. At the same time, the desire to pursue spiritual life like art, has become stronger, and the imagination and creativity of people has been further stimulated. Besides, driven by the Internet science and technology, many scenes that were considered incredible previously can already be presented to people with great power and novelty, which in turn has become the driving force and foundation of the imagination and creativity of people and promotes human development and progress.

Science and technology is the primary productive force, by virtue of the innovation of technology like the computer, the digitization of special effect has become possible and has developed continuously to meet the demand for film and television of people, promoting the prosperity of film and television in China. It can be said that the development of special effect is not only the progress of science and technology, but also integrates the contents that can reflect characteristics of the times such as the thinking of people and social reality. The study on the digital application of special effect is of great significance for researches of film and television.

\section{The analysis on value of digital application of special effect}

Speaking of the digitization of special effect, people cannot help thinking of international films like Avatar and Captain America, which makes so many film fans go crazy. When watching these films, people are usually attracted by the plot, and no one doubts its authenticity, which is the wonderful point of digital processing technology of special effect. The progress of computer processing technology has improved the digital application and popularization of special effect and the processing technology of sound and pictures has also been upgraded. At present, people are already able to simulate various real sounds according to the requirements of the plot, and the visual processing is more realistic. The value of digital application of film and television mainly demonstrates as follows. First of all, from the perspective of investment in film and television, the production of special effect can make up for the film and television effects that cannot or hard to be realized in the reality, meeting the sensory requirements of the audience and saving the costs of film and television production to a certain extent; Secondly, from the perspective of actors, in many films and television dramas, the plot is generally fictional and there are always many dangerous scenes such as death or explosion for actors to perform according to the requirements of the plot. Whereas, the digital processing of special effect can not only reach desired effect of production, but also ensure the safety of actors; Thirdly, from the perspective of production performance of film and 
television, the application of digital processing of special effect is able to make the plot more vivid and present scene effect with higher quality so as to bring rich sensory experiences to the audience and improve the comprehensive quality and proficiency of film and television works; Fourthly, form the perspective of expression of emotion in film and television, technology serves for the creation, and the digitization of special effect makes all kinds of scenes as much as possible be produced. The production of visual and audio special effect increases the authenticity of pictures, which undoubtedly makes the overall production more alive and vivid, and the whole story more appealing so as to highlight the main idea of works.

\section{The digitization of special effect}

\subsection{Digital processing of scene atmosphere}

When designing scenes of film and television, the reasonable layout of space and depth of field, and the flash, optical haze, reflective instruments and so on are able to create the desired effect, and play an important role in the scene atmosphere. Except ZEMAX, CODE V is most widely used in so many optical special effect software. Over the years, users worldwide have successfully developed a large number of camera lens, microobjective, optical spectrum instrument, space optical system, laser scanning system, holographic display system, infrared imaging system, ultraviolet lithography system and so on. Therefore, CODE V offers the unique function of "camera lens magic wand". Users simply input such parameters as the band, relative aperture, field of view and zoom ratio of the system for the system design, the software can find corresponding structure to be chosen in its own patent portfolio. With the aid of optical software, the light of the scene can be set to achieve the reasonable effect of heightening the scene so that the light can be naturally incorporated into the scene and the picture of film and television is presented to the audience in an authentic and reasonable manner. To be more specific, the advanced and powerful optical digital technology can not only ensure the quality of scene shooting, offer excellent feelings for audience, but also integrate perfectly objects and scenes to produce the authenticity of pictures so as to stimulate the sense of trust from audience and strengthen the appeal of works; The application of aureole and glow in right angle can determine the overall shape of objects; The application of lens flare can effectively eliminate image deviation in real shooting and present contents more authentically. In a word, the designing and application of optical special effect in scenes can present objects more authentically and clearly, which is of great importance for desired atmosphere in film and television scenes.

\subsection{The digital application of particle effects in film and television production}

In the production of film and television, related particles can conduct the directional combination according to the requirements with digital processing software like $\mathrm{AE}$ and ParticleIIllusion, and particulate matter in scenes can conduct the slight alteration with the field force, which is the production of rain and fog special effect usually seen in the film and television works. However, the movement of particles is greatly affected by the environment, even small changes in the environment may change the particle movement, so if particle movement is the target, it is necessary to establish a more stable environment to ensure that particles can move freely in scenes designed to achieve the desired special effect. In addition, it is required to set fixed application mode for tiny particles to ensure the task can be achieved successfully according to design effects. The author once in the post production, combined characteristics of pIllusion and some problems in the post production and solved troubles in the animation production.

\subsection{The digital painting effect in special effect}

Unlike regular production of special effect, there is not specific requirement or restriction of scene conditions for digital painting effect, therefore, its application is wider in the production of special effect. Painting effects include optical system special effects mode and particle system special effects mode in the actual special effect production, and painting effects are often used with 
the two modes. In special effect scenes, paintbrush tools are generally used to sketch simple curves, and the important special effect of paintbrush is the appropriate handling of colors. Whereas in specific production, it is inevitable to select objects to be sketched and create the editable mode, and in this way, various kinds of paintbrushes can be used to sketch objects effectively to be presented clearly in film and television.

\section{The digital special effect in visual design}

No matter how special effects are produced, it serves for the expression of emotion for the entire work. While visual presentation is an important bridge of between the film and television works and the audience. Therefore, in the setting of film and television scenes, digital processing technology can play an important role in enhancing expression and highlighting atmosphere so that the audience has authentic and rich experience, and the interaction with film and television works is deepened. With the development of film industry, this digital special effect technology is recognized more and more widely.

\subsection{The digital processing of shot scenes}

There are always many memorable scenes in film and television works. With the aid of computer digital technology, the sense of depth and quality of pictures can be improved and complex character relationship and story plot can be presented clearly to the audience. Besides, by virtue of light and shadow, the entire picture is more appealing so that the audience feels like in film and television scenes. In general, shot scenes are restricted by many elements and one of key elements is the effective design of macroscopic scene. What's more, the coordination of the former and latter scene cannot be ignored. It is the coordination of various elements that ensure effectively uniform shots. Specifically, it is important to ensure the sense of depth of pictures in mobile shooting.

\subsection{The influence of film and television atmosphere on visual design}

The two sensory organs which have the greatest influence on the emotion of the audience are eyes and ears and the production of the film and television works are mainly conducted from both visual and audio perspectives. In order to obtain a richer and more authentic emotional experience, there are higher requirements for the music and light in scene setting, which are also important basis for special effect technology. On the one hand, it is known that light is of significantly importance to film and television scenes even for people who are not experts in the industry. The environment is usually created to serve the story plot, while the light adjustment enhances the rendering, so that the scene can better reflect the mood of characters and resonate with the audience. Different light have different effects. Dark, bright, dazzling light with different colors and the length of ray of light are needed to be set correspondingly according to the requirements of plot to create appropriate atmosphere. On the other hand, music mainly affects auditory system of people. There are different types of music to express different emotion and mood. And different music match with different scenes and it is in indispensable element in film and television works. Audio engineer choose different music for different plots, on the one hand, it can improve the charm of works and attracting the attention of the audience. On the other hand, audience can predict unknown scene and plot according to types of music, which, to some extent, improves the film watching effect of audience.

\subsection{The authenticity of film and television production}

Art originates from life, and any creation is based on a certain truth. Therefore, authenticity is one of characteristics of art creation. At the same time, art is higher than life. Art mirrors the creator's comprehension and creation of life, which is also the charm of works, but it is undeniable that this sublimation still depends on the reality. Because the audience and the creators of film and television works are human, living in real life, of course, the audience and creators have imagination and creativity, which is their commonplace. Therefore, many people dream through film and television works. And this kind of dream is an ingenious combination of reality and hope. 
There is no doubt that the use of digital technology will enrich the dream, and works will be enriched with more tension and attraction. However, does the production of special effects can be divorced from reality? The answer is no. Just imagine a person riding a horse in the woods, usually by virtue of computer-generated technology, that is, conduct the cutout processing to characters, then synthesize with corresponding scenes, but the audience can easily determine the scene is not real, which will affect the feeling of the plot, and even the entire work will have a negative impact. We have always seen lots of good shots in film and television works spreading through the Internet, which is harmful to the healthy development of the industry. Therefore, in the creation of special effect, we must also uphold the principle of authenticity, which is the respect for the work and for the audience. In this way, emotion that the work want to express can be conveyed reasonably to the audience. In addition to the requirements of technology, the shooting team and creation team need to have a serious working attitude to ensure the quality of film and television works.

\section{Conclusion}

In summary, the development of computer processing technology have promoted the updating of the digital technology of special effect, the development of film and television, and enriched the film watching experience of audience. It can be said that special effect in the film industry is more and more important. However, at the same time, many creators have received enormous economic benefits brought by special effect, thus focusing on the special effect while neglecting the plot setting, making works be out of content and lack of cultural connotation. Therefore, it must also be clearly recognized that special effect technology serves for the film and television works, but cannot be overwhelming, which requires all film and television creators pragmatic and clear special effect technology serves the content of film and television, and establish the correct outlook of value and benefit, strive to create more meaningful works for audience, promoting the development of China's film and television industry.

\section{References}

[1] Gao Ziying. The Analysis on the Digital Application of Special Effect in Visual Design[J]. Consumer Electronics Magazine, 2013.

[2] Hou Yanjun. Digital Application of Special Effect in Visual Design[J]. Journal of News Research, 2014, 5 (8).

[3] Yu Xiaoni. Study on the Digital Application of Special Effect in Visual Design[J]. Electronics World, 2014. 